$16^{\text {th }}$ International Congress of Metrology, 04010 (2013)

DOI: $10.1051 /$ metrology/201304010

(c) Owned by the authors, published by EDP Sciences, 2013

\title{
Novel mathematical and statistical approaches to uncertainty evaluation: introducing a new EMRP research project
}

\author{
Markus Bär ${ }^{1}$, Clemens Elster ${ }^{1}$, Sebastian Heidenreich ${ }^{1}$, Clare Matthews ${ }^{2, a}$, Leslie Pendrill ${ }^{3}$ and Louise Wright ${ }^{2}$ \\ ${ }^{1}$ Physikalisch-Technische Bundesanstalt (PTB), Abbestr. 2-12, 10587 Berlin, Germany \\ ${ }^{2}$ National Physical Laboratory (NPL), Hampton Road, Teddington TW11 0LW, UK \\ ${ }^{3}$ Sveriges Tekniska Forskningsinstitut (SP), Brinellgatan 4, SE-504 62 Borås, Sweden
}

\begin{abstract}
The European Metrology Research Programme (EMRP) is funding project EMRP-NEW04 on novel mathematical and statistical approaches to uncertainty evaluation. Technical areas covered by the project include uncertainty evaluation for inverse problems, regression, computationally expensive models, and decision-making and conformity assessment. Here we describe the work to be carried out in each technical area, including an introduction to the application case-studies used within the project.
\end{abstract}

\section{Introduction}

Measurement uncertainty evaluation is at the foundation of metrology. Without it measurement results cannot be compared, either among themselves or with reference values given in a specification or standard. The foundation of traceability is the propagation of uncertainties from primary standards provided by national metrology institutes (NMIs) to end users. Unreliable evaluation of uncertainties can have a huge negative economic impact.

Dissemination of measurement results, reliable decision-making and conformity assessment require harmonised treatment of uncertainty. The current state of the art for uncertainty evaluation in metrology is provided by the Joint Committee for Guides in Metrology (JCGM) Guide to the expression of uncertainty in measurement (GUM) [1] and its Supplements [2, 3]. In addition, a guide to the role of measurement uncertainty in decisionmaking and conformity assessment has recently been published by the JCGM [4]. Existing guidelines are successfully applied across many areas of metrology, but they do not cover some of the new challenges arising from modern measurement systems. Examples occur in biochemistry, transport processes, regulation within industry and applications with multivariate measurement. New mathematical and statistical methods are required to address uncertainty evaluation in these rapidly growing metrology applications.

The European Metrology Research Programme (EMRP) is funding a three-year project, EMRP-NEW04, on novel mathematical and statistical approaches to uncertainty evaluation. The project involves nine European NMIs and focuses on methods for evaluation of

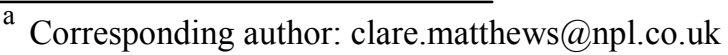

uncertainty in the context of (1) regression and inverse problems, (2) computationally expensive systems, and (3) conformity assessment and decision-making. Methods developed will be illustrated via a selection of case studies on ELISA (enzyme-linked immunosorbent assays), PCR (polymerase chain reaction), flow, thermophysical applications, scatterometry, healthcare products and fire engineering. Here we introduce each of the technical areas, including their relevant case studies, outline the key issues and aims of the work and describe initial results from the project as applied to scatterometry.

\section{Inverse problems and regression}

The GUM guidelines for uncertainty evaluation are based on the assumption of a suitable model that relates the quantity of interest (the measurand) to the influence quantities. However, in many applications it is not the quantity of interest that is measured but some other output parameter from which the quantity of interest is deduced and this requires the solution of inverse problems. Approximate solutions may be obtained by the application of least-squares approaches with subsequent linearisation for the evaluation of uncertainty, but this is often inadequate for nonlinear models.

Within the project we are investigating the use of Bayesian statistics to provide reliable and harmonised procedures for problems involving regression and inverse problems. The methods being developed allow inclusion of prior knowledge and physical constraints, errors in both stimulus and response variables, and quantification of the sensitivity of the results obtained to the assumptions made. Methods will be demonstrated using analysis of ELISA and PCR data, inference of 
thermophysical properties using the laser flash technique, and determination of calibration curves in flow meters.

Further details of this work are given in the related paper: Novel mathematical and statistical approaches to uncertainty evaluation in the context of regression and inverse problems [4].

\section{Computationally expensive systems}

Many important applications in metrology are described by model equations whose numerical solutions are computationally expensive. The longer a model takes to solve using a given computer and piece of software, the more computationally expensive the model is. Most of these computationally expensive systems are also strongly nonlinear, meaning that linear approximations for uncertainty evaluation may provide invalid results. Supplement 1 to the GUM recommends simulations using a Monte Carlo method. However, current Monte Carlo methods are based on a large number of model evaluations and consequently are not practical for computationally expensive systems due to the time involved or the computational power requirements.

Using flow, material thermophysical properties and scatterometry as case studies, we are exploring two approaches for uncertainty evaluation in the context of computationally expensive systems. The first is to develop smart sampling methods, of which importance sampling [e.g. 5], Latin hypercube sampling [e.g. 6] and polynomial chaos [e.g. 7] have been identified as candidates for investigation. The second involves the use of surrogate models that are easier to evaluate than the original physical models. Within the project, response surface methodology [8] and Gaussian process emulation [e.g. 9] will be explored. Polynomial chaos can also be applied to surrogate models and here we will investigate an approach using polynomial chaos expansion coefficients.

One area of metrology to which we are applying these methods is scatterometry. In scatterometry, inverse methods are used to reconstruct grating geometries of photo masks from measured diffraction efficiencies (see Figure 1) $[10,11]$. The efficiency is a measure of the power of the diffracted light in relation to that of the incident light beam. The finite element methods (FEM) typically used to numerically simulate the diffraction patterns are computationally expensive as they require solution of Maxwell's equations. It is therefore not practical to apply Markov Chain Monte Carlo methods within Bayesian inference. To resolve this issue, we will replace the FEM-based forward model by a surrogate model that is substantially less computationally expensive. The surrogate model is based on polynomial chaos expansion of the prior distribution of grating geometry parameters.

\section{light beam} efficiencies
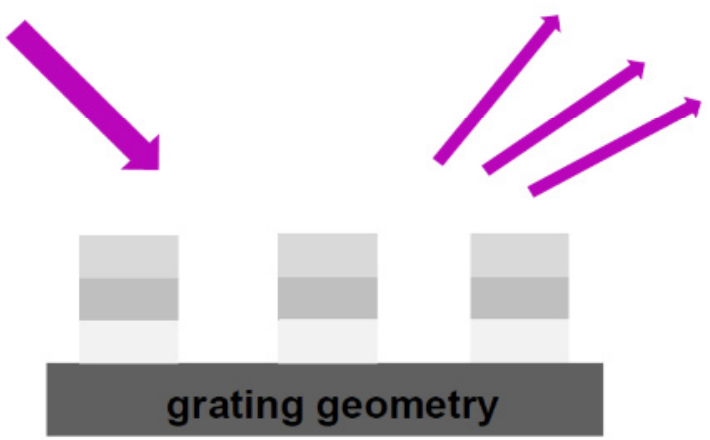

/ II

Figure. 1 Sketch of the scatterometry set up. Refracted light efficiencies are used to reconstruct the grating geometry.

The expansion coefficients defining the surrogate model are calculated by projection integrals of the full forward model onto the chosen polynomial basis. For the integration we choose specific, training points where the full model has to be calculated rigorously with FEM.

We chose a uniform prior distribution for three geometry parameters and constructed a surrogate model with the polynomial chaos expansion. Figure 2 shows the comparison of a rigorous FEM calculation and the surrogate approximation using the same geometry parameters. The surrogate model produces a small loss of accuracy and a reduction of run time of two orders of magnitude. The relative differences between the calculations of efficiency of the surrogate model and the full FEM model are less than $1 \%$ and the surrogate model has a run time of less than a second where the full FEM model takes several minutes to solve.

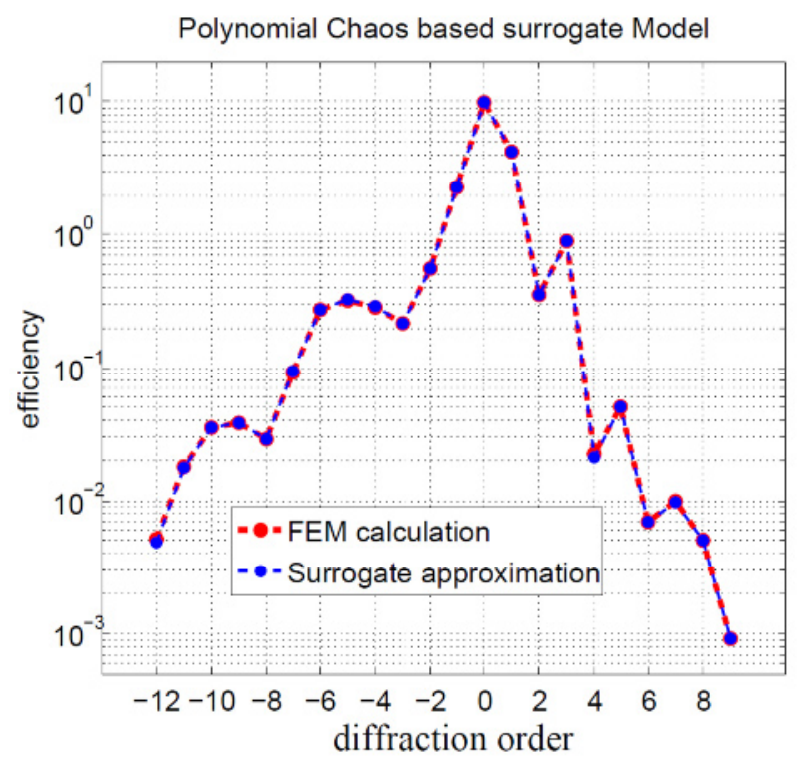

Figure. 2 Simulated scatterogram for an extreme ultraviolet lithography mask. Scattering efficiency is calculated both with a finite element solver (red) and a polynomial chaos-based surrogate model (blue). 
We will test the surrogate model for different grating geometries and apply the relevant concepts developed elsewhere within the project to employ a Bayesian approach to solve the inverse problem (i.e., estimating the grating dimensions from the efficiency measurements).

\section{Conformity assessment and decision- making}

Many measurements are made to provide basis for decisions about a product or a process. An unnecessarily high risk exists of incorrect decisions without reliable measurement uncertainty analysis. Many applications need guidance for conformity assessment which goes beyond current guides and standards (e.g. [12, 13, 14]).

Where conformity assessment of a product, process or system relies on measurement as a principal source of information, measurement uncertainty can lead to incorrect estimates of the consequences of product error, and increase the risk of making incorrect decisions. There is increasing appreciation of the fact that better understanding of the role of measurement uncertainty in conformity assessment will aid the metrologist in setting a 'fit-for-purpose' level of measurement effort prior to performing a given task. This ability will in turn support increased, mutual understanding between the metrologist and those ordering such tasks, about the significance and limitations of the measurements when making decisions of conformance.

With reference to case studies on fire engineering and healthcare products, the project will develop tools and guidelines for reliable decision-making and conformity assessment, with particular emphasis on the multivariate case. Early work has focused on establishing the state-ofthe-art for measurement uncertainty in conformity assessment. There is currently no single or standard approach applied, with decision rules differing depending on the product or process being tested and the nature of the measurement required (e.g. [14]). Advancement in the field requires clear separation of measurement dispersion and product variation. Technical work within the scope of the EMRP-NEW04 will focus on the use of economic analysis as a methodology within conformity assessment and metrology, and multivariate conformity assessment, where several attributes vary simultaneously, as this is not currently addressed in guidelines and standards.

\section{Summary}

The aim of the EMRP-NEW04 project is to provide guidance on methods for uncertainty evaluation in the context of regression, inverse and computationallyexpensive problems and conformity assessment. Here we have given a brief outline of the technical work to be covered and introduced the specific applications that will be considered as case studies.

The case studies will be used to provide training material and software. Results from each case study will also be extended to create generic guidance material for best-practice in each of the technical areas. These bestpractice guides will be aimed at NMI metrology and industrial end users and will be available electronically via the project website.

\section{Acknowledgement}

This work is part of EMRP-project NEW 04 "Novel mathematical and statistical approaches to uncertainty evaluation“. The EMRP is jointly funded by the EMRP participating countries within EURAMET and the European Union.

\section{References}

1. BIPM, IEC, IFCC, ILAC, ISO, IUPAC, IUPAP and OIML (2008). Evaluation of measurement data - Guide to the expression of uncertainty in measurement. Joint Committee for Guides in Metrology, JCGM 100:2008

2. BIPM, IEC, IFCC, ILAC, ISO, IUPAC, IUPAP and OIML (2008). Evaluation of measurement data Supplement 1 to the Guide to the expression of uncertainty in measurement - Propagation of distributions using a Monte Carlo method. Joint Committee for Guides in Metrology, JCGM 101:2008 3. BIPM, IEC, IFCC, ILAC, ISO, IUPAC, IUPAP and OIML (2011). Evaluation of measurement data Supplement to the Guide to the expression of uncertainty in measurement - Extension to any number of output quantities. Joint Committee for Guides in Metrology, JCGM 102:2011

4. C. Elster, K. Klauenberg, M. Bär, A. Allard, N. Fischer, G. Kok, A. van der Veen, P. Harris, M. Cox, I. Smith, L. Wright, S. Cowen, P. Wilson, S. Ellison, Novel mathematical and statistical approaches to uncertainty evaluation in the context of regression and inverse problems, $16^{\text {th }}$ International Congress of Metrology (2013)

5. R. Y. Rubinstein, D. P. Kroese, Simulation and the Monte Carlo method, Wiley (2008)

6. J. C. Helton, F. J. Davis, Reliab. Eng. Syst. Safe. 81, 23-69 (2003)

7. D. Xiu, Commun. Comput. Phys. 5 (2-4), 242-272 (2009)

8. R. H. Myers, D. C. Montgomery, C. M. AndersonCook, Response surface methodology, Wiley, New Jersey (2009)

9. A. O’Hagan, Reliab. Eng. Syst. Safe. 91, 1290-1300 (2006)

10. H. Gross, M.-A. Henn, A. Rathsfeld, M. Bär, Stochastic modelling aspects for an improved solution of the inverse problem in scatterometry, in Advanced Mathematical \& Computational Tools in Metrology and Testing IX, Series on Advances in Mathematics for Applied Sciences 84, World Scientific New Jersey (2012) 11. M. -A. Henn, H. Gross, F. Scholze, M. Wurm, C. Elster, M. Bär, Opt. Express 20, 12771-12786 (2012) 12. BIPM, IEC, IFCC, ILAC, ISO, IUPAC, IUPAP and OIML (2012). Evaluation of measurement data - The role of measurement uncertainty in Conformity 
Assessment. Joint Committee for Guides in Metrology, JCGM 106:2012

13. ISO 10576-1 (2003). Statistical methods Guidelines for the evaluation of conformity with specified requirements.

14. AFNOR (2004). Use of uncertainty in measurement: presentation of some examples and common practices. French Standardization, FD x07-022. 